\section{Cancer Council looks to a healthy future}

Continued from page 2

\section{DIMINISHING SUFFERING FROM CANCER \\ Patient and family support}

In February 1986 the Council established a committee under the late Dr Fred Gunz which was to set the agenda for activities in the previously relatively neglected area of patient and family support. The principal objectives of this initiative were to

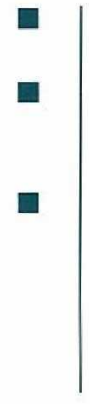

give patients a voice in their care, in the sense of discovering what they saw their needs to be; provide information to cancer patients about their condition and its treatment in language virtually everyone could understand; and do something to remedy known deficiencies in their care, notably the lack of accommodation near cancer centres for patients forced to travel long distances for daily treatment and an under resourced palliative care system in need of expansion and additional trained staff.

Today, as a result of a partnership between the Council and a major Sydney teaching hospital, one hostel - the 28-bedroom Casuarina Lodge - stands within the grounds of Westmead Hospital. Soon, as the result of another such partnership, a second hostel - the 37-bedroom Blue Gum Lodge - will be built in the grounds of Greenwich Hospital to accommodate patients being treated at nearby Royal North Shore Hospital. The Council has also introduced fellowships to enable doctors and nurses to further their training in palliative care overseas and brings distinguished visitors in palliative care in medicine to Sydney to share knowledge with local colleagues.

The Council provides a number of other services to cancer patients and their families including a telephone counselling service and live-in carer crisis service.

\section{FUTURE PLANS}

The Council now has regional offices in Lismore, Newcastle and Wollongong and with local community support and participation will be reaching out to more country centres. Much of the impetus came from the Council's merchandise program which, since 1990, has drawn thousands of people into its orbit.

The Council's Act is being updated, the major change being to establish a board with expertise across its program areas rather than statutory representation of specific organisations. Greater use will be made of experts on a needs basis rather than relying on the many standing committees of professionals which presently exist. The board will continue to be composed of nine members (Dr George Rubin is Deputy Chair, having succeeded Dr Sue Morey at the beginning of 1992).

A Memorandum of Understanding was signed at the end of November with the Health Department whereby the Council, with funding from the Department, will undertake a number of programs on its behalf according to agreed performance criteria. The Memorandum will set the pattern of collaboration between the Department and the Council.

Elaine Henry

Executive Director, NSW Cancer Council
INFECTIOUS DISEASES

\section{MEASLES}

Six hundred and seventy-two measles notifications were received for 1992 . The notification rate for NSW is 11.3 notifications per 100,000 population. Orana and Far West Region notified 75 cases of measles for a rate of 53.4 notifications per 100,000 population.

Of the 672 cases 87 ( 12.9 per cent) were less than one year of age and 269 (27.0 per cent) were less than five years of age. All children should be routinely offered measles-mumps-rubella vaccine at 12 months of age.

\section{RUBELLA}

For 1992, 243 notifications have been received for rubella. The notification rate for NSW is 4.1 per 100,000 population. Hunter Area notified 55 cases of rubella for a notification rate of 11.2 notifications per 100,000 population.

Of the 243 cases 4 (1.6 per cent) were less than one year of age and 92 (37.8 per cent) males aged 15 to 24 .

\section{ARBOVIRUS INFECTIONS}

The heavy late spring rains and flooding in the Upper Murray have reached the far western area and all backwaters and billabongs are at high levels. As the water levels recede small pools will remain to provide an enormous number of potential breeding sites for the inland vector of arboviral disease Culex annulirostris. This could not have occurred at a worse time as the population of this mosquito explodes from late December through to February. With all the additional water to act as breeding sites the potential for very high population densities is likely.

There has also been an increase in the population of birdlife, native and feral animals that may act as the natural host for arboviruses. What this means in practical terms is that this year there may be an increase in Ross River virus infection (one of the arboviruses that can cause infection in human).

Alphavirus (this includes Ross River virus) infections are driven by three climatic factors: high rainfall, flooding and tidal inundation. Large parts of inland NSW have experienced both high rainfall and flooding while on the coast inundation is a regular occurrence. Depending on temperature, mosquito population densities and alphavirus activity, Ross River virus infection rates may be higher than usual.

There are a number of simple steps to take to avoid being bitten by a mosquito:

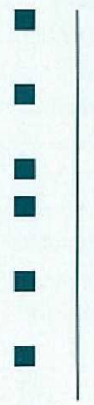

wear loose fitting long sleeved shirts and trousers; apply insect repellent containing DEET to those parts of the body exposed to biting;

lightly spray clothing with repellent; ensure insect screens are in good condition (don't forget the chimney);

if there are no strong winds hang mosquito coils; and

Terry Carvan kill mosquitoes by swatting, spraying or using mats impregnated with insecticide.

Senior Environmental Health Officer, South West Region. 


\section{TABLE 1}

INFECTIOUS DISEASE

NOTIFICATIONS BY HEALTH AREA

AND REGION CUMULATIVE1992

\begin{tabular}{|c|c|c|c|c|c|c|c|c|c|c|c|c|c|c|c|c|c|c|c|}
\hline Condition & CSA & SSA & ESA & sws & WSA & WEN & NSA & CCA & ILL & HUN & NCR & NER & OFR & CWR & SWR & SER & OTH & $\mathrm{U} / \mathrm{K}$ & TOTAL \\
\hline Adverse event after immunisation & 3 & 3 & $=$ & - & 2 & - & - & 1 & - & 1 & 5 & 7 & 1 & 1 & 2 & 5 & - & - & 205 \\
\hline AIDS & 43 & 6 & 25 & 5 & 21 & 8 & 34 & 10 & 6 & 14 & 15 & 6 & 1 & 3 & 5 & 3 & - & - & 205 \\
\hline Arboviral infection & - & - & - & - & 1 & - & - & 2 & - & - & - & 2 & - & - & - & - & - & - & 5 \\
\hline Ross River fever & 2 & 2 & - & - & 6 & 6 & 6 & 5 & 8 & 24 & 112 & 32 & 60 & 10 & 25 & 1 & - & - & 299 \\
\hline Other arboviruses & - & - & - & - & - & - & 1 & - & - & - & 3 & 1 & - & - & 3 & - & - & - & 8 \\
\hline Brucellosis & - & - & - & 1 & - & - & - & - & - & - & - & - & - & - & - & - & - & - & 1 \\
\hline Foodborne illness (NOS) & 8 & 4 & 33 & 10 & 65 & 21 & - & 30 & 10 & 7 & 5 & 5 & 36 & 1 & 1 & 2 & - & - & 238 \\
\hline Gastroenteritis (instit.) & 57 & 6 & 9 & 28 & 10 & 1 & 1 & 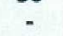 & 1 & 94 & 2 & 96 & 16 & - & - & 97 & - & - & 418 \\
\hline Gonorrhoea infection & 71 & 29 & 165 & 25 & 26 & 1 & 25 & 9 & 3 & 14 & 23 & 13 & 19 & 15 & 8 & 9 & - & - & 455 \\
\hline $\mathrm{H}$ influenzae epiglottitis & $=$ & 5 & 1 & 3 & 7 & 3 & 4 & - & 3 & 7 & 6 & 6 & - & - & 1 & 4 & - & - & 50 \\
\hline $\mathrm{H}$ influenzae meningitis & 5 & 6 & 4 & 6 & 6 & 8 & 18 & 4 & 8 & 10 & 6 & 5 & 2 & 4 & 5 & 7 & - & - & 104 \\
\hline H influenzae septicaemia & - & 2 & 2 & 5 & 2 & - & 3 & - & - & 5 & 1 & - & - & 2 & 1 & 1 & - & - & 24 \\
\hline $\mathrm{H}$ influenzae infection (NOS) 3 & 3 & 2 & 2 & 1 & 2 & - & 2 & 6 & 3 & 2 & - & 3 & 1 & 2 & 2 & 5 & - & - & 36 \\
\hline Hepatitis A - acute viral & 95 & 48 & 119 & 37 & 51 & 12 & 91 & 8 & 32 & 30 & 134 & 127 & 92 & 13 & 12 & 11 & 1 & - & 913 \\
\hline Hepatitis B - acute viral & 5 & 4 & 30 & 6 & 5 & 5 & 4 & 3 & 6 & 1 & 9 & 5 & 21 & 2 & 3 & 2 & - & - & 111 \\
\hline Hepatitis B - unspecified & 429 & 435 & 21 & 756 & 422 & 34 & 338 & 37 & 29 & 128 & 64 & 48 & 31 & 25 & 14 & 29 & 2 & - & 2842 \\
\hline Hepatitis C - acute viral & 1 & 1 & 4 & 1 & 10 & 1 & 3 & 1 & 3 & - & 8 & 6 & 4 & 3 & - & 2 & - & - & 48 \\
\hline Hepatitis C - unspecified & 584 & 212 & 425 & 247 & 348 & 76 & 274 & 370 & 86 & 448 & 573 & 73 & 12 & 60 & 25 & 41 & 1 & - & 3855 \\
\hline Hepatitis D - unspecified & -. & - & 1 & - & - & 1 & - & 1 & - & 3 & - & - & - & - & - & - & - & - & 7 \\
\hline Hepatitis, acute viral (NOS) & - & - & 2 & 2 & 4 & - & - & 1 & - & - & - & 2 & 3 & 2 & 1 & - & - & - & 17 \\
\hline HIV infection* & 69 & 24 & 221 & 16 & 30 & 8 & 37 & 8 & 3 & 26 & 16 & - & 3 & 3 & 3 & 6 & - & 195 & 688 \\
\hline Hydatid disease & - & 1 & - & - & - & - & - & - & - & - & 1 & 2 & - & 1 & - & - & - & - & 5 \\
\hline Legionnaires' Disease & 6 & 2 & 2 & 36 & 17 & 2 & 4 & 8 & 2 & 2 & 2 & - & - & - & - & 1 & - & - & 84 \\
\hline Leprosy & - & - & - & 1 & 1 & 1 & - & - & - & - & - & 1 & - & - & 1 & - & - & - & 5 \\
\hline Leptospirosis & - & 1 & - & - & - & 1 & - & - & - & - & 6 & 2 & - & 5 & 1 & - & - & - & 16 \\
\hline Listeriosis & - & 2 & - & 2 & - & 2 & 5 & 1 & - & 1 & 1 & - & - & 1 & - & - & - & - & 15 \\
\hline Malaria & 10 & 7 & 8 & 4 & 15 & $=$ & 23 & 2 & 8 & 4 & 8 & 7 & 1 & 1 & 5 & 3 & - & - & 106 \\
\hline Measles & 54 & 82 & 9 & 127 & 57 & 31 & 29 & 11 & 13 & 100 & 30 & 32 & 75 & 7 & 4 & 11 & - & - & 672 \\
\hline Meningococcal meningitis & 6 & 7 & - & 6 & 7 & 5 & 1 & 6 & 6 & 9 & 9 & 5 & 2 & 10 & - & 4 & - & - & 83 \\
\hline Meningococcal septicaemia & 2 & 1 & 2 & 3 & - & 2 & - & 3 & 1 & 1 & - & - & 2 & - & - & - & - & - & 17 \\
\hline Meningococcal infection (NOS) & - & - & 2 & - & - & - & 1 & - & 1 & 1 & - & 4 & 3 & 2 & - & - & - & - & 14 \\
\hline Mumps & - & - & 4 & 2 & 3 & - & 1 & - & 2 & 4 & 1 & - & - & - & 2 & 1 & - & - & 20 \\
\hline Mycobacterial atypical & 56 & 30 & 42 & 20 & 29 & 5 & 36 & 1 & 13 & 23 & 5 & 3 & 2 & - & 3 & 2 & - & - & 270 \\
\hline Mycobacterial tuberculosis & 53 & 47 & 27 & 69 & 55 & 6 & 53 & 17 & 14 & 6 & 12 & 6 & $\overline{1}$ & 5 & 8 & 6 & 1 & - & 386 \\
\hline Mycobacterial infection (NOS) & 13 & 3 & 4 & 1 & 5 & 3 & 7 & 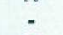 & 5 & 6 & - & 1 & - & - & 1 & - & - & - & 49 \\
\hline Pertussis & 7 & 11 & 7 & 11 & 18 & 14 & 27 & 10 & 5 & 14 & 29 & 3 & - & 1 & 1 & 7 & - & - & 165 \\
\hline Q Fever & - & - & $=$ & - & 9 & 5 & - & 1 & 1 & 8 & 75 & 32 & 37 & 10 & 4 & 2 & - & - & 184 \\
\hline Rubella & 5 & 8 & 18 & 7 & 30 & 9 & 31 & 13 & 3 & 55 & 15 & 25 & 3 & 2 & 3 & 16 & - & - & 243 \\
\hline Salmonella bovis morbificans & 1 & 3 & 1 & - & 2 & 1 & 2 & - & - & 3 & 1 & 1 & - & $=$ & - & 1 & - & - & 16 \\
\hline Salmonella typhimurium & 10 & 25 . & 2 & 25 & 31 & 19 & 23 & 10 & 10 & 21 & 2 & 3 & 6 & - & 7 & - & - & - & 194 \\
\hline Salmonella (NOS) & 24 & 38 & 40 & 48 & 38 & 29 & 85 & 17 & 9 & 32 & 52 & 30 & 24 & 23 & 14 & 17 & - & - & 520 \\
\hline Syphilis infection & 134 & 45 & 130 & 58 & 38 & 10 & 43 & 2 & 8 & 17 & 108 & 48 & 120 & 17 & 13 & 2 & 1 & - & 794 \\
\hline Tetanus & - & - & - & 1 & - & - & - & - & - & - & - & - & - & - & - & - & 1 & - & 2 \\
\hline Typhoid and paratyphoid & 4 & 2 & 6 & 1 & 3 & - & 5 & - & 1 & - & - & - & - & - & 2 & - & - & - & 24 \\
\hline
\end{tabular}

\section{TABLE 2}

VACCINE PREVENTABLE DISEASE NOTIFICATIONS

BY HEALTH AREA AND REGION

CUMULATIVE 1992

$\begin{array}{lll}\text { Condition } & \text { CSA SSA ESA SWSWSA WEN NSA CCA ILL HUN NCR NER OFR CWR SWR SER OTH TOTAL }\end{array}$

$\begin{array}{lccccccccccccccccccc}\text { Measles } & 54 & 82 & 9 & 127 & 57 & 31 & 29 & 11 & 13 & 100 & 30 & 32 & 75 & 7 & 4 & 11 & - & 672 \\ \text { Mumps } & - & - & 4 & 2 & 3 & - & 1 & - & 2 & 4 & 1 & - & - & - & 2 & 1 & - & 20 \\ \text { Pertussis } & 7 & 11 & 7 & 11 & 18 & 14 & 27 & 10 & 5 & 14 & 29 & 3 & - & 1 & 1 & 7 & - & 165 \\ \text { Rubella } & 5 & 8 & 18 & 7 & 30 & 9 & 31 & 13 & 3 & 55 & 15 & 25 & 3 & 2 & 3 & 16 & - & 243 \\ \text { Tetanus } & - & - & - & 1 & - & - & - & - & - & - & - & - & - & - & - & 1 & - & & 2\end{array}$

\section{TABLE 3}

RARELY NOTIFIED INFECTIOUS DISEASES

BY HEALTH AREA AND REGION

CUMULATIVE 1992

Condition

CSA SSA ESA SWSWSA WEN NSA CCA ILL HUN NCR NER OFR CWR SWR SER OTH

TOTAL

Brucellosis

Hydatid Disease

Leprosy

Leptospirosis

Listeriosis 


\section{TABLE 4}

SUMMARY OF NSW INFECTIOUS

DISEASE NOTIFICATIONS

DECEMBER 1992

\begin{tabular}{|c|c|c|c|c|}
\hline \multirow{3}{*}{ Condition } & \multicolumn{4}{|c|}{ Number of cases notified } \\
\hline & \multicolumn{2}{|c|}{ Period } & \multicolumn{2}{|c|}{ Cumulative } \\
\hline & December 1991 & December 1992 & December 1991 & December 1992 \\
\hline Adverse reaction & 4 & & 4 & 31 \\
\hline AIDS & 31 & $\overline{6}$ & 368 & 205 \\
\hline Arboviral infection & 3 & 1 & 476 & 312 \\
\hline Brucellosis & - & - & 2 & 1 \\
\hline Cholera & - & - & - & - \\
\hline Diptheria & - & - & - & - \\
\hline Foodborne illness (NOS) & 105 & 7 & 2861 & 238 \\
\hline Gastroenteritis (instit.) & 58 & 1 & 138 & 418 \\
\hline Gonorrhoea & 27 & 5 & 415 & 455 \\
\hline $\mathrm{H}$ influenzae epiglottitis & 4 & 4 & 25 & 50 \\
\hline $\mathrm{H}$ influenzae $\mathrm{B}$ - meningitis & 5 & 4 & 64 & 104 \\
\hline H influenzae B - septicaemia & 2 & 1 & 12 & 24 \\
\hline $\mathrm{H}$ influenzae infection (NOS) & 6 & - & 125 & 36 \\
\hline Hepatitis A & 81 & 7 & 1100 & 913 \\
\hline Hepatitis B & 127 & 23 & 1385 & 2953 \\
\hline Hepatitis C & 161 & 44 & 800 & 3903 \\
\hline Hepatitis D & N/A & - & N/A & 7 \\
\hline HIV infection* & 69 & 60 & 774 & 668 \\
\hline Hydatid disease & - & - & 7 & 5 \\
\hline Legionnaires' Disease & 3 & - & 29 & 84 \\
\hline Leprosy & - & - & - & 5 \\
\hline Leptospirosis & 1 & 1 & 32 & 16 \\
\hline Listeriosis & N/A & $i$ & N/A & 15 \\
\hline Malaria & 17 & 1 & 202 & 106 \\
\hline Measles & 72 & 35 & 432 & 672 \\
\hline Meningococcal meningitis & 8 & 4 & 56 & 83 \\
\hline Meningococcal septicaemia & 4 & - & 19 & 17 \\
\hline Meningococcal infection (NOS) & 3 & 1 & 42 & 14 \\
\hline Mumps & N/A & - & N/A & 20 \\
\hline Mycobacterial tuberculosis & 36 & 4 & 352 & 386 \\
\hline Mycobacterial - atypical & 10 & 2 & 114 & 270 \\
\hline Mycobacterial infection (NOS) & 5 & $\overline{1}$ & 163 & 49 \\
\hline Pertussis & 4 & 4 & 48 & 165 \\
\hline Plague & - & - & - & - \\
\hline Poliomyelitis & - & - & - & - \\
\hline Q Fever & 13 & 1 & 185 & 184 \\
\hline Rubella & 5 & 8 & 61 & 243 \\
\hline Salmonella infection (NOS) & 95 & 13 & 1274 & 730 \\
\hline Syphilis & 61 & 7 & 622 & 794 \\
\hline Tetanus & 1 & - & 6 & 2 \\
\hline Typhoid and paratyphoid & 1 & - & 61 & 24 \\
\hline Typhus & - & - & - & - \\
\hline Viral haemorrhagic fevers & - & - & - & - \\
\hline Yellow fever & - & - & - & - \\
\hline
\end{tabular}

*Data to November only

\section{TABLE 5}

NOTIFICATIONS OF NON-NOTIFIABLE SEXUALLY

TRANSMITTED INFECTIONS FROM SEXUAL

HEALTH CLINICS JANUARY-DECEMBER 1992

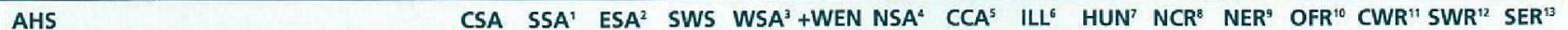
Infection

Chlamydia trachomatis

Donovanosis

Genital herpes

Genital warts

Non-specific urethritis

Lymphogranuloma

$\begin{array}{ccccccccccccccc}- & 8 & 157 & - & 44 & 5 & 3 & 15 & 40 & 2 & 6 & 7 & - & - & - \\ - & - & - & - & - & - & - & - & - & - & - & - & - & - & - \\ - & 12 & 406 & - & 53 & 18 & 6 & 29 & 50 & 1 & 9 & 14 & - & - & - \\ - & 105 & 907 & - & 260 & 56 & 8 & 191 & 159 & 18 & 21 & 10 & - & - & - \\ - & 9 & 577 & - & 274 & 26 & 1 & 70 & 68 & 5 & 8 & 5 & - & - & - \\ - & - & - & - & - & - & - & - & - & - & - & - & - & - & -\end{array}$


TABLE 6

INFECTIOUS DISEASE

NOTIFICATIONS BY

MONTH OF ONSET

\begin{tabular}{|c|c|c|c|c|c|c|c|c|c|c|c|c|c|}
\hline Condition & Jan & Feb & Mar & Apr & May & Jun & Jul & Aug & Sep & Oct & Nov & Dec & Total \\
\hline Adverse reaction & 4 & 8 & 3 & 1 & 6 & 2 & - & 4 & 2 & - & 1 & - & 31 \\
\hline AIDS & 25 & 14 & 17 & 17 & 24 & 18 & 21 & 20 & 12 & 13 & 18 & 6 & 205 \\
\hline Arboviral infection & - & $=$ & 2 & 1 & - & - & - & - & $=$ & 2 & - & - & 5 \\
\hline Ross River fever & 14 & 40 & 85 & 77 & 39 & 10 & 11 & 7 & 5 & 6 & 4 & 1 & 299 \\
\hline Other arboviruses & - & - & 2 & - & - & 1 & - & - & - & 2 & 3 & - & 8 \\
\hline Brucellosis & - & - & - & - & - & 1 & - & - & - & - & - & $=$ & 1 \\
\hline Foodborne illness (NOS) & 55 & 28 & 27 & 20 & 15 & 7 & 13 & 18 & 20 & 13 & 15 & 7 & 238 \\
\hline Gastroenteritis (instit.) & 88 & 7 & 17 & 9 & 36 & 22 & 41 & 161 & 9 & 23 & 4 & 1 & 418 \\
\hline Gonorrhoea infection & 31 & 22 & 49 & 38 & 49 & 31 & 56 & 42 & 52 & 40 & 40 & 5 & 455 \\
\hline $\mathrm{H}$ influenzae epiglottitis & 4 & 1 & 3 & 2 & 4 & 10 & 4 & 4 & 4 & 5 & 5 & 4 & 50 \\
\hline $\mathrm{H}$ influenzae meningitis & 5 & 9 & 10 & 5 & 11 & 13 & 9 & 13 & 9 & 11 & 5 & 4 & 104 \\
\hline H influenzae septicaemia & 1 & 1 & 3 & 3 & 3 & 2 & 5 & - & 3 & 1 & 1 & 1 & 24 \\
\hline $\mathrm{H}$ influenzae infection (NOS) & 5 & 2 & 1 & 2 & 2 & 4 & 5 & 6 & 1 & 3 & 5 & - & 36 \\
\hline Hepatitis A - acute viral & 114 & 98 & 121 & 98 & 90 & 83 & 66 & 66 & 53 & 78 & 39 & 7 & 913 \\
\hline Hepatitis B - acute viral & 10 & 12 & 18 & 21 & 18 & 9 & 5 & 5 & 10 & 3 & - & - & 111 \\
\hline Hepatitis B - unspecified & 280 & 179 & 274 & 253 & 247 & 321 & 291 & 286 & 253 & 253 & 182 & 23 & 2842 \\
\hline Hepatitis C - acute viral & 14 & 7 & 3 & 5 & 6 & 2 & 4 & 1 & 4 & 2 & - & - & 48 \\
\hline Hepatitis C - unspecified & 235 & 256 & 316 & 254 & 450 & 400 & 432 & 417 & 339 & 412 & 300 & 44 & 3855 \\
\hline Hepatitis D - unspecified & 1 & - & - & 1 & 3 & - & - & - & $=$ & 1 & 1 & - & 7 \\
\hline Hepatitis, acute viral (NOS) & - & 3 & 1 & 4 & 2 & 1 & 1 & - & 1 & 2 & 2 & - & 17 \\
\hline HIV infection * & 95 & 74 & 72 & 60 & 72 & 52 & 56 & 45 & 37 & 45 & 58 & N/A & 668 \\
\hline Hydatid disease & 2 & - & 2 & - & - & 1 & - & - & - & - & - & - & 5 \\
\hline Legionnaires' Disease & $\overline{1}$ & 9 & 3 & 42 & 8 & 5 & 8 & 3 & 1 & 2 & 2 & - & 84 \\
\hline Leprosy & 1 & 1 & - & - & 1 & 1 & 1 & - & - & - & - & - & 5 \\
\hline Leptospirosis & 3 & 2 & - & 1 & 4 & 1 & 2 & 1 & 1 & - & - & 1 & 16 \\
\hline Listeriosis & 1 & 1 & 1 & 3 & 1 & 1 & 1 & - & 3 & 2 & - & 1 & 15 \\
\hline Malaria & 12 & 5 & 16 & 9 & 14 & 17 & 13 & 8 & 7 & 3 & 1 & 1 & 106 \\
\hline Measles & 48 & 31 & 34 & 22 & 41 & 31 & 22 & 26 & 61 & 109 & 212 & 35 & 672 \\
\hline Meningococcal meningitis & - & 3 & 2 & 8 & 2 & 6 & 16 & 13 & 9 & 14 & 6 & 4 & 83 \\
\hline Meningococcal septicaemia & 1 & - & $=$ & $=$ & - & 2 & 2 & 3 & 3 & 1 & 5 & - & 17 \\
\hline Meningococcal infection (NOS) & 2 & 2 & - & - & $=$ & - & 2 & 3 & 1 & 1 & 2 & 1 & 14 \\
\hline Mumps & 3 & $\overline{5}$ & 2 & - & 3 & 2 & $\overrightarrow{1}$ & 1 & 2 & - & 1 & - & 20 \\
\hline Mycobacterial atypical & 33 & 32 & 48 & 25 & 31 & 31 & 23 & 17 & 22 & 3 & 3 & 2 & 270 \\
\hline Mycobacterial tuberculosis & 77 & 33 & 36 & 38 & 30 & 40 & 21 & 32 & 29 & 22 & 24 & 4 & 386 \\
\hline Mycobacterial infection (NOS) & 7 & 5 & 7 & 2 & 3 & 6 & - & 2 & 3 & 6 & 7 & 1 & 49 \\
\hline Pertussis & 5 & 15 & 25 & 7 & 6 & 9 & 13 & 10 & 18 & 22 & 31 & 4 & 165 \\
\hline Q Fever & 13 & 12 & 11 & 13 & 9 & 22 & 21 & 28 & 20 & 22 & 12 & 1 & 184 \\
\hline Rubella & 6 & 7 & 7 & 4 & 1 & 1 & 5 & 14 & 35 & 72 & 83 & 8 & 243 \\
\hline Salmonella bovis morbificans & 1 & 1 & 1 & 2 & 3 & 1 & - & 2 & 1 & 1 & 3 & - & 16 \\
\hline Salmonella typhimurium & 20 & 21 & 51 & 23 & 23 & 7 & 9 & 10 & 10 & 15 & 5 & $=$ & 194 \\
\hline Salmonella (NOS) & 99 & 59 & 57 & 52 & 41 & 33 & 37 & 42 & 24 & 32 & 31 & 13 & 520 \\
\hline Syphilis infection & 54 & 85 & 70 & 83 & 88 & 95 & 89 & 77 & 51 & 53 & 42 & 7 & 794 \\
\hline Tetanus & 1 & - & - & - & - & - & - & - & 1 & - & - & - & 2 \\
\hline Typhoid and paratyphoid & 6 & 4 & 2 & - & 3 & 2 & 3 & 2 & 1 & - & 1 & - & 24 \\
\hline
\end{tabular}

*Data to November only

\section{TABLE 7}

VACCINE PREVENTABLE

DISEASE NOTIFICATIONS BY MONTH OF ONSET

\begin{tabular}{|c|c|c|c|c|c|c|c|c|c|c|c|c|c|}
\hline Condition & Jan & Feb & Mar & Apr & May & Jun & Jul & Aug & Sep & Oct & Nov & Dec & Total \\
\hline Measles & 48 & 31 & 34 & 22 & 41 & 31 & 22 & 26 & 61 & 109 & 212 & 35 & 672 \\
\hline Mumps & 3 & 5 & 2 & - & 3 & 2 & 1 & 1 & 2 & - & 1 & - & 20 \\
\hline Pertussis & 5 & 15 & 25 & 7 & 6 & 9 & 13 & 10 & 18 & 22 & 31 & 4 & 165 \\
\hline Rubella & 6 & 7 & 7 & 4 & 1 & 1 & 5 & 14 & 35 & 72 & 83 & 8 & 243 \\
\hline Tetanus & 1 & - & - & - & $=$ & - & - & - & 1 & - & - & - & 2 \\
\hline
\end{tabular}

Abbreviations used in this Bulletin:

CSA Central Sydney Health Area, SSA Southern Sydney Health Area, ESA Eastern Sydney Health Area, WSA Western Sydney Health Area, WEN Wentworth Health Area, NSA Northern Sydney Health Area, CCA Central Coast Health Area, ILL Illawarra Health Area, HUN Hunter Health Area, NCR North Coast Health Region, NER New England Health Region, OFR Orana \& Far West Health Region, CWR Central West Health Region, SWR South West Health Region, SER South East Health Region, OTH Interstate/Overseas, U/K Unknown, NOS Not Otherwise Stated.

Please note that the data contained in this Bulletin are provisional and subject to change because of late reports or changes in case classification. Data are tabulated where possible by area of residence and by the disease onset date and not simply the date of notification or receipt of such notification. 